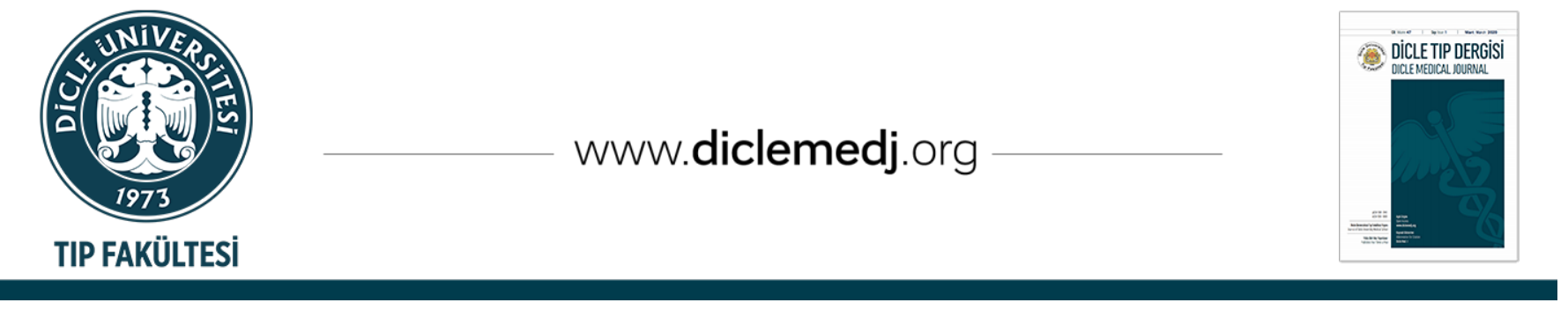

Özgün Araștırma / Original Article

\title{
Hemodiyaliz Hastalarında Kateter İlişkili Kan Dolaşım Enfeksiyonlarının Değerlendirilmesi
}

\author{
Enver Yüksel iD 1, Şafak Kaya iD 2, Emrah Günay iD 1 \\ 1 Diyarbakır Gaziyaşargil Eğitim ve Araştırma Hastanesi Nefroloji Bölümü Diyarbakır, Türkiye \\ 2 Diyarbakır Gaziyaşargil Eğitim ve Araştırma Hastanesi Enfeksiyon hastalıkları Bölümü Diyarbakır, Türkiye
}

Geliş: 11.01.2020; Revizyon: 17.07.2020; Kabul Tarihi: 21.07.2020

$\ddot{0} \mathbf{z}$

Amaç: Bu çalışmanın amacı, hemodiyaliz hastalarında kateter ilişkili kan dolaşımı enfeksiyonlarını (KIKDE) incelemektir.

Yöntemler: Çalışmamızda hastanemizde 1 Ocak 2013 ve 1 Nisan 2019 tarihleri arasında kateter ilişkili kan dolaşım enfeksiyonları gelișen hastaların verileri retrospektif olarak değerlendirildi. Çalışmaya 18 yaş üstü, kan ve kateter kültürlerinde aynı mikroorganizma üremesi saptanan hastalar alındı. 18 yaş altı, kan ve kateter kültürlerinde farklı mikroorganizma üremesi saptanan hastalar çalışmaya alınmadı. Çalışma kriterlerini karşılayan 65 hastadan 109 kateter ilişkili kan dolaşımı enfeksiyonu vakası alındı.

Bulgular: Çalışmaya 109 kateter iliş̧ili kan dolaşım enfeksiyonları epizodu alındı. Kan kültürlerine bakıldığında 10'unda $(\% 9,2)$ Klebsiella pneumoniae, 9'unda $(\% 8,3)$ Escherichia coli, 23 'ünde $(\% 21,1)$ metisiline dirençli koagülaz-negatif stafilokok (MRKNS), 1'isinde $(\% 0,9)$ metisiline duyarlı koagülaz negatif stafilokok (MSKNS), 15 'inde $(\% 13,8)$ metisiline dirençli Staphylococcus aureus (MRSA), 23'ünde (\%21,1) metisiline duyarlı Staphylococcus aureus (MSSA), 5'inde $(\% 4,6)$ Acinetobacter spp, 7 'sinde $(\% 6,4)$ Enterobacter spp., 5'inde $(\% 4,6)$ Streptococcus spp., 6'sinda $(\% 5,5)$ Enterococcus faecalis, 2'sinde $(\% 1,8)$ Stenotrophomonas maltophilia, 2 'sinde $(\% 1,82)$ Pseudomonas aeruginosa, ve 1'inde $(\% 0,9)$ Morganellamorgani üremesi saptandı.

Sonuç: Hemodiyaliz hastalarında ciddi morbidite ve mortaliteye yol açan KİKDE'nin tedavisi hayati önem arz etmektedir. Bu nedenle her hastane ve diyaliz merkezleri kendi etken dağılımlarını bilmelidir. Böylece kültür sonuçları çıkana kadar başlanacak olan ampirik tedavi seçiminde uluslararası kılavuzlara ek olarak merkez verileri de değerlendirilebilir.

Anahtar kelimeler: Hemodiyaliz, kateter ilişkili kan dolaşım enfeksiyonları, santral venöz kateter.

\section{DOI: 10.5798/dicletip.800259}

Correspondence / Yazışma Adresi: Enver Yüksel, Diyarbakır Gaziyaşargil Eğitim ve Araştırma Hastanesi Nefroloji Bölümü Diyarbakır, Türkiye e-mail : ferhat_enver@hotmail.com 


\title{
Evaluation Of Catheter-Related Bloodstream Infections In Hemodialysis Patients
}

\begin{abstract}
Objective: The purposes of this study was to investigate catheter-related bloodstream infections (CRBSI) in hemodialysis patients.

Methods: Our study was planned as a retrospective study. Patients who developed catheter-related bloodstream infections between January 2013 and april 2019 were included. The study included patients over the age of 18 who had the same microorganisms in their blood and catheter cultures. Patients who were under 18 years of age with different blood and blood cultures were excluded from the study. 109 episodes of catheter-related blood circulation infections were taken from 65 patients who met the study criteria.
\end{abstract}

Results: In total, we identified 109 episodes of catheter-related bloodstream infections. Ten of them caused by Klebsiella pneumoniae, 9 were caused by Escherichia coli, 24 were caused by Staphylococcus, including 23 by methicillin-resistant coagulase negative Staphylococcus and 1 by methicillin-sensitive coagulase negative Staphylococcus , 28 were caused by staphylococcus aureus, including 15 by methicillin resistance staphylococcus aureus and 23 methicillin sensitive Staphylococcus aureus, 5 them caused by Acinetobacter sp., 7 of them caused by Enterobacter sp., 5 of them caused by Streptococcus sp., 6 of them caused by Enterococcus faecalis, 2 of them caused by Stenotrophomonas maltophilia, 2 of them caused by Pseudomonas aeruginosa, and 1of them caused by Morganella morgani.

Conclusion: As a result, treatment of catheter-related bloodstream infections, which leads to serious morbidity and mortality in hemodialysis patients, is vital. For this reason, every hospital and dialysis centers should know their own distribution of agents. Thus, in addition to international guidelines, center data can be evaluated in the selection of empirical treatment to be initiated until culture results are obtained

Keywords: Hemodialysis, catheter-related bloodstream infections, central venous catheter.

\section{GíRiş}

Kronik böbrek yetmezliği gelişen hastalarda son dönem böbrek yetmezliği tedavi seçeneklerinden biri hemodiyalizdir. Türk Nefroloji Derneği 2017 kaylt sistemine göre 77311 renal replasman tedavisi gören hastanın 58635'i hemodiyalize girmektedir ${ }^{1}$. Arteriovenöz fistül (AVF) en çok tavsiye edilen vasküler giriş yoludur ${ }^{2}$. Arterio-venöz fistül şansı olmayan veya acil hemodiyaliz tedavisi gereken hastalar tercihen santral venöz kateter (SVK) takılarak hemodiyalize alınmaktadır. Uygulama yeri olarak subklavian, juguler veya femoral venler seçilebilir ${ }^{3,4}$. Tünelsiz SVK' lerin kateter ilişkili kan dolaşım enfeksiyonları (KİKDE) için daha riskli olduğu kabul edilmektedir. $\mathrm{Bu}$ enfeksiyonlar hastalarda mortalite artışıly beraber endokardit ve artrit gibi diğer komplikasyonların da artışına sebep olmaktadır ${ }^{5-7}$.
Santral venöz kateterlerin diğer damar erişim yollarına kıyasla avantajları olmasına rağmen en önemli dezavantajı KİKDE gelişimidir. Kan dolaşım enfeksiyonları yapılan bir çalışmada $\% 12$ 'lik sıklıkla tüm hastane enfeksiyonları içinde en sık karşılaşılan dördüncü (\%12) enfeksiyon olarak bildirilmiştir ${ }^{8}$. Hastane kaynaklı kan dolaşım enfeksiyonlarının \%85'inin SVK'ye bağlı olduğu bildirilmektedir ${ }^{4,6,9}$.

Kateter ilişsili kan dolaşım enfeksiyonlarında en sık görülen etkenler koagülaz negatif stafilokok (KNS), Staphylococcus aureus, Enterococ sp. ve Candida türleridir ${ }^{10}$. Gramnegatif basiller KIKDE'lerin \%20-50'sini oluşturmaktadır 11-13. Yapılan çalışmalarda KİKDE'lerin çoğunun yeterli önlemler alındığında engellenebildiği gösterilmiştir ${ }^{14}$.

Üremik tablo, yetersiz beslenme, komorbid hastalıklar, kateterin süresi, kateter yeri, yetersiz kateter bakımı, kateterin acil olarak 
takılması, femoral venin kullanılması, ve sağlık çalışanlarından kaynaklanan bulaş gibi bir çok faktör bu enfeksiyonların ortaya çıkmasını kolaylaștırabilir ${ }^{15,16}$. Ayrıca enfeksiyon gelişimine katkıda bulunabilecek bir diğer durum da kateter yerleştirilirken asepsi kurallarına uyulmamasıdır. Bu nedenle kateter takılırken el hijyeni, cilt hazırlığı, maksimum steril bariyer önlemleri, uygun yerleştirme bölgesi seçimi gibi uluslararası önerilere kesinlikle uymak gerekir ${ }^{15-16}$.

\section{YÖNTEMLER}

$\mathrm{Bu}$ çalışma hastanemiz etik kurulunun 25/10/2019 tarihli ve 358 sayılı kararı alındıktan sonra retrospektif olarak planlandı. Çalışmamız 1 Ocak 2013-1 Nisan 2019 tarihleri arasinda hastanemiz veri sistemi taranarak son 6 yllda hemodiyalize giren, kateter ve perifer kan kültürlerinde üreme saptanan hastalar alınmıştır. Çalışma için belirlenen verilerde eksiklik saptanan, kateter kültürlerinde ve kan kültürlerinde farklı mikroorganizma üremesi saptanan ve 18 yaş altı hastalar çalıșma dıșı bırakıldı. Çalışmaya 75 hastaya ait $124 \mathrm{KIKKDE}$ epizodu alındı. Üç hastanın yaşının 18 yaşından küçük olması, 3 hastanın kan ve kültür örneklerindeki üremelerinde farklı mikroorganizmaların saptanması ve 2 hastada da kültür antibiyogramlarına ulaşılamaması nedeniyle çalışma dışında bırakıldı. Çalışmaya 67 hastaya ait 109 KİKDE epizodu alındı. Kateter örneklerinin ekimleri tekniklerine uygun olarak yapılmış olup kültürler için BACTEC 9120 (Becton Dickinson, ABD) sistemi kullanılmıştır. Antibiyotik duyarlılık testleri "Clinical and Laboratory Standards Institute" kriterlerine uygun olarak disk difüzyon yöntemi ile yapılmıștır.

\section{BULGULAR}

Çalışmamıza 109 KİKDE epizodu alındı. Hastaların yaş ortalaması 58,6 $\pm 21,8$ (19-99), 44'ü $(\% 65,7)$ kadın, 23'ü (\%33.3) erkek idi. Bu hastaların 58'inde $(\% 89,9)$ juguler kateter, 6'sinda $(\% 7,3)$ femoral kateter ve 3'ünde $(\% 3,6)$ subklavian kateter mevcut idi. Hastaların komorbid durumlarına bakıldığında 27'si (\%40) diyabetes mellitus, 44'ü $(\% 65,6)$ hipertansiyon, 7'si $(\% 10,4)$ koroner arter hastalığı, 5'i $(\% 7,4)$ kronik obstrüktif akciğer hastalı̆̆ı, 7'si $(\% 10,4)$ serebrovasküler olay geçirmiş ve 1 'i $(\% 1,4)$ immunsupresif ilaçlar kullanıyordu.

Kan kültürü üremelerin incelendiğinde 10 'unda $(\% 9,2)$ Klebsiella pneumoniae, 9'unda $(\% 8,3)$ Escherichia coli, 23'ünde (\%21,1) MRKNS, 1 'inde $(\% 0,9)$ MSKNS, 15 'inde $(\% 13,8)$ metisiline dirençli Staphylococcus aureus (MRSA), 23'ünde $(\% 21,1)$ metisiline duyarlı Staphylococcus aureus (MSSA), 5'inde $(\% 4,6)$ Acinetobacter spp., 7'sinde $(\% 6,4)$ Enterobacter spp., 5'inde $(\% 4,6)$ Streptococcus spp., 6'sinda (\%5,5) Enterococcus faecalis, 2'sinde $(\% 1,8)$ Stenotrophomonas maltophilia, 2'sinde $\quad(\% 1,82) \quad$ Pseudomonas aeruginosa, ve 1 'inde $(\% 0,9)$ Morganella morgani üremesi saptandı (șekil:1). Hastaların kateter yerine göre üreyen mikroorganizmalar ise tablo $1^{\prime}$ de özetlenmiştir.

Tablo I: Kateter yerine göre kültürde üreyen etkenler

\begin{tabular}{|c|c|c|c|c|}
\hline Mikroorganizmalar & juguler & femoral & subklavian & total \\
\hline Klebsiella pneumoniae & 10 & & & 10 \\
\hline Escherichia coli & 6 & 3 & & 9 \\
\hline MRSA & 14 & & 1 & 15 \\
\hline MSSA & 21 & 1 & 1 & 23 \\
\hline MRKNS & 21 & 1 & 1 & 23 \\
\hline MSKNS & 1 & & & 1 \\
\hline Enterobacter sp. & 5 & 2 & & 7 \\
\hline Acinetobacter sp. & 5 & & & 5 \\
\hline $\begin{array}{l}\text { Stenotrophomonas } \\
\text { maltophilia }\end{array}$ & 2 & & & 2 \\
\hline Streptococcus sp. & 5 & & & 5 \\
\hline Enterococcus faecalis & 5 & 1 & & 6 \\
\hline Pseudomonas aeruginosa & 2 & & & 2 \\
\hline Morganella morgani & 1 & & & 1 \\
\hline
\end{tabular}

MRSA: Metisiline dirençli Staphylococcus Aureus, MSSA: Metisiline duyarlı Staphylococcus Aureus, MRKNS: Metisiline dirençli koagülaz-negatif stafilokok, MSKNS: Metisiline duyarlı koagülaz-negatif stafilokok 


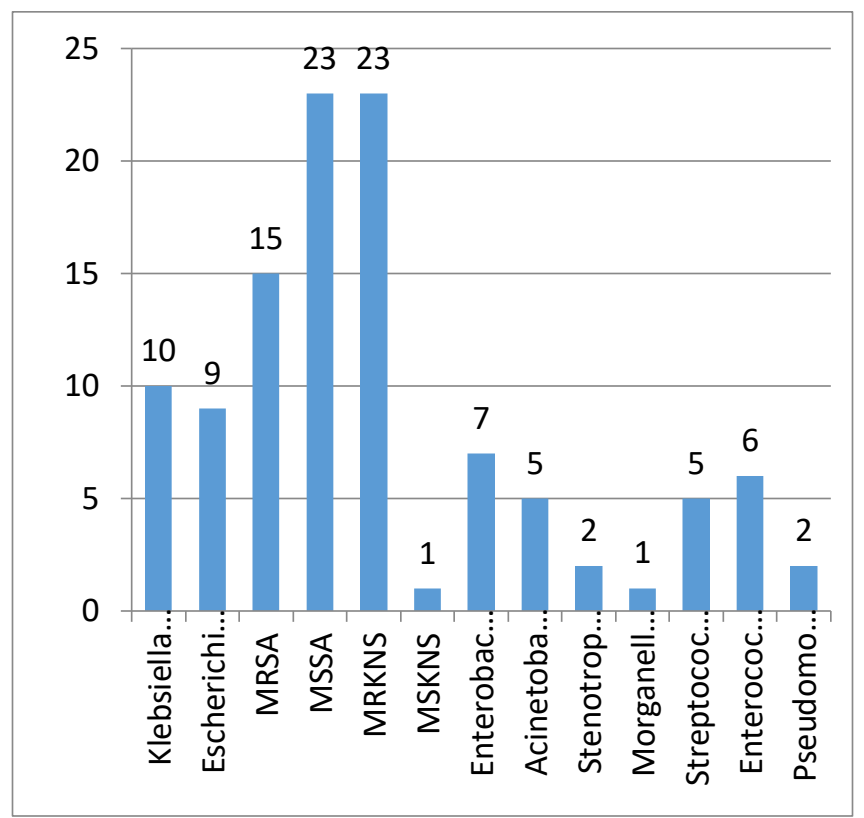

MRSA: Metisiline dirençli Staphylococcus Aureus, MSSA: Metisiline duyarl Staphylococcus Aureus, MRKNS: Metisiline dirençli koagülaz-negatif stafilokok, MSKNS: Metisiline duyarlı koagülaz-negatif stafilokok

Şekil 1: Kateter ilişkili kan dolaşım enfeksiyonlarında üreyen mikroorganizmalar

\section{TARTIŞMA}

Hemodiyaliz tedavisi gören kronik böbrek yetmezliği hastalarının bağışıklık sistemleri, zayıf olduğu için enfeksiyona eğilimlidirler. Hastalar ya AVF yoluyla yada SVK takılarak hemodiyalize alınmalıdırlar. Santral venöz kateterler, sağlı bakımıyla ilişkili bakteriyemilerin en önemli nedenlerinden biri olup mortalite oranı \%20-40 arasında değișmektedir ${ }^{17}$. $\mathrm{Bu}$ nedenle enfeksiyon oranları, etken mikroorganizmalar, olası risk faktörleri ve antibiyotik duyarlılıklarının bilinmesi ampirik tedavinin belirlenmesi ve mortalitenin azaltılması için önemlidir ${ }^{18}$.

Hemodiyalize giren hastalarda enfeksiyon açısından en güvenilir damar yolu AVF iken en riskli olan femoral kateterlerdir. Zhang ve arkadaşlarının, 1160 hastadan oluşan 17 yıllık verilerin incelendiği retrospektif bir çalışmada femoral ven yerleşimli kateterler, KİKDE için anlamlı risk faktörü olarak bulunmuştur ${ }^{19}$. Kidney Disease Improving Global Outcomes klavuzunun ilk sırada önerdiği SVK yeri juguler kateterlerdir. Özellikle kalıcı juguler kateterler, diyalize giren hastalarda tromboz gibi komplikasyonlar açısından subklavian kateterlere göre daha güvenilir bulunmuștur ${ }^{20}$. Bizim hastalarımızda femoral ve subklavian kateter kullanımı daha azdı. Hastanemizde 140 rutin hemodiyaliz hastasinda yalnızca 1'i $(\% 0,7)$ kalıcı femoral kateterle hemodiyalize alınmaktaydı. Bu yüzden femoral ve subklavian kateter ilişkili enfeksiyonlar değerlendirilememiştir.

Kateter ilişkili kan dolaşım enfeksiyonlarının risk faktörleri incelendiğinde hastaya ait faktörler katetere bağlı risk faktörlerinden daha önemlidir ${ }^{21}$ İmmünsupresyon (malignite, nötropeni), uzun süreli total parenteral nütrisyon kulanımı, kronik böbrek yetmezliği ve diyabetes mellitus gibi komorbit durumlar KIKKDE gelişimini kolaylaştırmaktadır ${ }^{21}$. Hastalarımızdan biri immunsüpresif tedavi kullanırken 27'si (\%40) diabetes mellitus hastası idi. SVK'lerin kalış süresi de enfeksiyon gelişimi açısından oldukça önemlidir. Hemodiyaliz hastalarında önerilen SVK'lerin en kısa süre çıkarılmasıdır.

Hastanelerde damar içi kateterler (DİK), hemodiyaliz tedavisinin yanı sıra hem medikal tedavide hemde nutrisyonel destek sağlamak için kullanılmaktadır ${ }^{22}$. Amerika Birleşik Devletlerinde Marschall ve arkadaşlarının yaptığı çalışmada, KİKDE olan 42 olgunun \%57'sinde gram-pozitif bakteriler, \%17'sinde gram-negatif bakteriler ve \%14'ünden Candida türleri izole edilmiştir ${ }^{23}$. Stafilokoklar (Staphylococcus epidermidis ve Staphylococcus aureus), DİK ile ilişkili kan dolaşımı enfeksiyonlarında en sık karşılaşılan etkenler olarak kabul edilse de Enterococcus ve Candida da sıklıkla karşımıza çıkmaktadır ${ }^{6,24}$. Ülkemizde yapılan bir diğer çalışmada ise KİKDE olarak değerlendirilen olgularda en sık Acinetobacter spp. saptanırken KNS ikinci sırayı almıștır ${ }^{18}$. Bizim çalışmamız spesifik bir hasta grubu olan hemodiyaliz hastaları ile sinırlı olup sadece 
SVK'sı olan ve KİKDE saptanan hastalar dahil edilmiştir. Çalışmamızda KÍKDE'de en çok saptanan mikroorganizma Staphylococcus aureus olup bunların 15'i $(\% 39,4)$ MRSA idi. İkinci sıklıkta koagulaz negatif stafilokoklar yer almakta idi. KIKDE saptanan koagulaz negatif stafilokokların 23'ü $\quad(\% 95,8)$ metisiline rezistans idi.

KíKDE'lerde kateterin çlkarılıp çıkarılmaması konusunda farklı görüşler vardır ${ }^{25-28}$. Lorente ve arkadaşlarının 2014 yılında yaptıkları çok merkezli prospektif çalışmada kateter infeksiyonu şüphesi olan hastalarda kateterin çıkarılmasının 30 günlük mortaliteyi azaltmadığı, bu nedenle her hastada önerilmediği bildirmektedir ${ }^{26}$. Öte yandan Jensen ve arkadaşlarına göre ise KIKDE'de kateterin çıkarılmaması kötü prognoza sebep olur ${ }^{27}$. Kateter çlkarılma kararı; etken mikroorganizmaya, tünel enfeksiyonun veya kateter giriş yeri enfeksiyonun olup olmamasına veya uygulanan tedaviye alınan yanıta göre karar verilir ${ }^{29}$. Tedavi süresi etkenin özelliğine, kateter çekilip çekilmemesine göre değişkenlik göstermektedir. KİKDE tanısı konulup tedavi kararı verildikten sonra sistemik antibiyotik başlanması esastır. Komplike olmayan hastalarda kateter çıkartıldıysa 5-7 gün, kateter çıkarılmadıysa 1014 gün antibiyotik tedavisine devam edilmesi önerilmektedir.

Sonuç olarak, hemodiyaliz hastalarında ciddi morbidite ve mortaliteye yol açan KIKNE'nin tedavisi hayati önem arz etmektedir. Bu nedenle enfeksiyon oranlarl, etken mikroorganizmalar, antibiyotik duyarlılıkları tedavinin etkinliği ve mortalitenin azaltılması açılarından önemlidir

Etik Kurul Kararı: Bu çalışma hastanemiz etik kurulunun 25/10/2019 tarihli ve 358 sayll kararı alındıktan sonra retrospektif olarak planlandi.
Çıkar Çatışması Beyanı: Yazarlar çıkar çatışması olmadığını bildirmişlerdir.

Finansal Destek: Bu çalışma her hangi bir fon tarafından desteklenmemiştir.

Declaration of Conflicting Interests: The authors declare that they have no conflict of interest.

Financial Disclosure: No financial support was received.

\section{KAYNAKLAR}

1. Türk Nefroloji Derneği 2017 yılı Türk böbrek kayıt sistemi raporu.

2. Miguel SS, Chow J. Vascular dialysis access flow measurement: early intervention through early detection. J Ren Care 2009; 35: 185-91.

3. Henderson DK, Mandell GL, Bennett JE, et al. Infections caused by percutaneous intravascular devices. Principles and Practice of Infectious Diseases. 6th ed. Philadelphia: Churchill-Livingstone 2005: 3347-62.

4. Ulusoy S. Hastane Enfeksiyonları ve Kontrolü Derneği, Damar İçi Kateter Enfeksiyonlarının Önlenmesi Çalışma Grubu. Damar içi kateter infeksiyonlarının önlenmesi kullanım kılavuzu. Hastane İnfeksiyonları Dergisi 2005; 9 (Ek 1): E3-32.

5. Ravani P, Palmer SC, Oliver MJ, et al. Associations between hemodialysis access type and clinical outcomes: a systematic review. J Am Soc Nephrol. 2013 [cited 2017 Jan 10]; 24: 465-73.

6. Brasil. Agencia Nacional de Vigilancia Sanitaria. Criterios diagnosticos relacionados a assistencia de saude. Serie: Seguranca do Paciente e Qualidade em Servicos de Saude 2017.

7. D'Amato-Palumbo S, Kaplan AA, Fein RS, et al. Retrospective study of microorganisms associated with vascular access infections in hemodialysis patients. Oral Surg Oral Med Oral Pathol Oral Radiol cited 2017 Jan 10; 115: 56-61.

8. Vincent JL, Bihari DJ, Suter PM, et al. The prevalence of nosocomial infection in intensive care units in Europe. Results of the European Prevalence of Infection in Intensive Care (EPIC) study. JAMA 1995; 274: 639-44. 
9. O'Grady NP, Alexander M, Dellinger EP, et al. Guidelines for the prevention of intravascular catheter-related infections. CID 2002; 35: 1281-307.

10. Wisplinghoff $H$, Bischoff $T$, Tallent SM, et al. Nosocomial bloodstream infections in US hospitals: analysis of 24,179 cases from a prospective nationwide surveillance study. Clin Infect Dis. 2004; 39: 309-17.

11. Gaynes R, Edwards JR; National Nosocomial Infections Surveillance System. Overview of nosocomial infections caused by gram-negative bacilli. Clin Infect Dis. 2005; 41: 848-54.

12. See I, Freifel AG, Magill SS. Causative organisms and associated antimicrobial resistance in healthcare-associated, central lineassociated bloodstream infections from oncology settings, 2009-2012. Clin Infect Dis. 2016; 62: 1203-9.

13. Aydın M, Kaşıkçıŏlu C, Nargiz Koşucu S, et al. Kan Dolaşımı İnfeksionu Etkenleri ve Antibiyotik Direnç Oranları KLIMIK Journal, vol. 29 no.2, 2016.

14. Yilmaz G, Koksal I, Aydin K, et al. Risk factors of catheter-related bloodstream infections in parenteral nutrition catheterization. JPEN J Parenter Enteral Nutr. 2007; 31: 284-7.

15. Grothe C, Belasco AGS, Bittencourt ARC, et al. Incidencia de infeccao da corrente sanguineanos pacientes submetidos a hemodialise por cateter venoso central. Rev Latino-Am 2010, vol.18, n.1, pp.73-80. ISSN 1518-8345.

16. Borges PRR, Bedendo J. Risk factors associated with temporary catheter-related infection in patients on dialysis treatment. Texto Contexto Enferm 2017 Jan 14; 24: 680-5.

17. Bouza E, Burillo A. Catheter related infections diagnosis and intravascular Treatment. Clin Microbiol Infect. 2002; 8: 265-74.

18. Aktaş E, Sarı EN, Seremet Keskin A, et al. Damar içi kateter ile ilişkili enfeksiyon etkenleri ve antibiyotik duyarlılıkları. Mikrobiyol Bül. 2011; 45: 86-92.
19. Zhang M, Xu Y, Jiang Z, et al. Study on risk factor of central venous catheter infection in ICU: 1160 patients report. 2017; 29: 1082-6.

20. Kdigo Clinical Practice Guideline For Chronic Kidney Disease 2012.

21. Gürsoy B, Gelecek S, Yorgancı K. Santral venöz kateter infeksiyonları. Yoğun Bakım Dergisi. 2006; 6: 196-203.

22. Safdar N, Mermel LA, Maki DG. The epidemiology of catheter-related infection in the critically Ill, pp: 1-22. Part of the Perspectives on Critical Care Infectious Diseases book series (CCID, volume 8).

23. Marschall J. Catheter-associated bloodstream infections: looking outside of the ICU. Am J Infect Control 2008; 36: S172.e5-8.

24. Pascual A. Pathogenesis of catheter-related infections: lessons for new designs. Clin Microbiol Infect 2002; 8: 256-64.

25. N.P. O'Grady and D. Pittet. Catheter-related infections in the critically ill kluwer academic publishers. new york, boston ... boston. (C2004 springer science + business media, inc. visit springer's ebookstore at.

26. Lorente L, Martín MM, Vidal P, et al. Working Group on Catheter Related Infection Suspicion Management of GTEIS/SEMICYUC. Should central venous catheter be systematically removed in patients with suspected catheter related infection? Crit Care. 2014; 18: 564.

27. Jensen $A G$, Wachmann $\mathrm{CH}$, Espersen $\mathrm{F}$, et al. Treatment and outcome of Staphylococcus aureus bacteremia: a prospective study of 278 cases. Arch Intern Med. 2002; 162: 25-32.

28. Tomar S, Lodha R, Das B, et al. Risk factors for central line associated bloodstream infections. Indian Pediatr. 2016; 53: 790-92

29. Mermel LA, Allon M, Bouza E, et al. Clinical practice guidelines for the diagnosis and management of intravascular catheterrelated infection: 2009 update by the Infectious Diseases Society of America. Clin Infect Dis. 2009; 49: 1-45. 